\title{
Study of Magnetic Fredericksz Transition in Ferronematics. Liquid Crystals Doped with Fine Magnetic Particles
}

\author{
P. Kopcansky, M. Koneracka, V. Zavisova, J. Jadzyn*, G. Czechowski* and B. Zywucki* \\ Institute of Experimental Physics, Slovak Academy of Sciences, Watsonova 47, 04353 Kosice, \\ Slovak Republic \\ * Institute of Molecular Physics, Polish Academy of Sciences, Smoluchowskiego 17, Poznan, Poland
}

\begin{abstract}
The magnetic Fredericksz transition in ferronematics (thermotropic nematic liquid crystals $7 \mathrm{CB}$ and MBBA combined with fine magnetic particles of size $10 \mathrm{~nm}$ ) as a function of concentration of magnetic particles was studied by using simple dielectric measurements. The increase of the threshold magnetic field is observed in 7CB based ferronematic while the decrease of threshold field in MBBA based ferronematic is observed when the concentration of magnetic particles is increased. Experimental results are disscussed in framework of Brochard, de Gennes and Burylov, Raikher theories.
\end{abstract}

\section{Introduction}

The combination of a liquid crystal and a magnetic fluid are so called ferronematic. Prior to the chemical synthesis of this material Brochard and de Gennes [1] constructed a continuum theory of magnetic suspension in liquid crystal. Rault et all [2] the basic magnetic properties of suspension of rod like $\gamma-\mathrm{Fe}_{2} \mathrm{O}_{3}$ particles in MBBA liquid crystal has studied. Burylov and Raikher [3,4] analysed the Brochard and de Gennes theory its limitations to real thermotropic systems applicability were given. The main difference between the above theories is that in thermotropic ferronematics with finite anchoring on particles the equilibrium orientational state is $\vec{n}(\vec{r}) \perp \vec{m}(\vec{r})$ (where $\vec{n}(\vec{r})$ is director of nematic molecule and $\vec{n}(\vec{r})$ is the local magnetization) not $\vec{n}(\vec{r}) \| \vec{m}(\vec{r})$ (the co-alignment postulate). The aim of this work is to prepare 7CB and MBBA based ferronematics to study the magnetic Fredericksz transition as a function of volume concentration of magnetic particles by means of simple dielectric measurements.

\section{Experimental results and discussions}

The magnetic particles had a mean diameter $D_{v}=10 \mathrm{~nm}$ and standard deviation $\sigma=0.325$. The ferronematics were prepared by adding the magnetic powder to liquid crystal and sonicating for 10 minutes. With reproducible measurements of Fredericks transition two samples of $7 \mathrm{C}: \mathrm{B}$ based ferronematics were obtained. The volume concentrations of magnetic particles were $f_{1}=2.5 \times 10^{-4}$ and $f_{2}=5 \times 10^{-4}$ respectively. The initial planar (7CB based ferronematic) and homeotropic (MBBA based ferronematic) alignment of nematic liquid crystal molecules were obtained by coating the capacitor electrodes with HTAB (hexadecyl tri methyl ammonium bromide). To orient the samples a $0.3 \mathrm{~T}$ magnetic field was used. The magnetic. Frederickz transition was indicated by dielectric measurements. The measured capacitor cell $(D=60 \mu \mathrm{m})$ was constructed from two flat thin $\mathrm{SnO}_{2}$ layer coated with non-magnetic glass electrodes which was connected to regulated thermostating system. The measurement of capacitance were carried out using standard RLC - bridge (an accuracy of $0.1 \%$ at a voltage $50 \mathrm{mV}$ and at a frequency $20 \mathrm{kHz}$ ). The temperature was stabilized with an accuracy $\pm 0.01^{\circ} \mathrm{C}$. A typical Fredericksz transition for MBBA based ferronematic with volume concentration $f=10^{-3}$ of magnetite is given in Fig. 1 . 


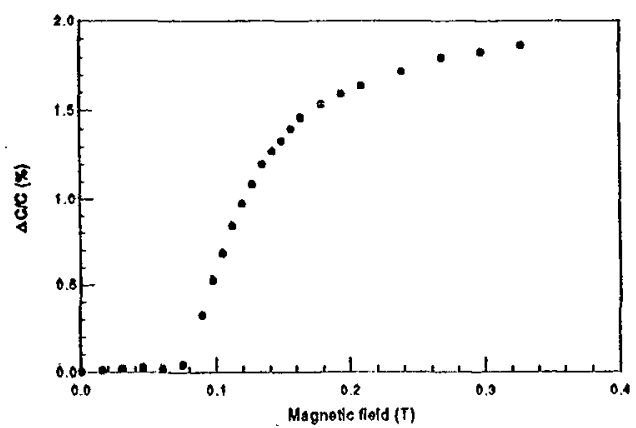

Figure 1: Typical Fredericksz transition in MBBA doped with volume concentration $f=10^{-3}$ of magnetic particles.

\begin{tabular}{cccc}
\hline $\mathrm{t}$ & \multicolumn{3}{c}{$\mu_{0} I_{C}[\mathrm{~T}]$} \\
\cline { 2 - 4 }$\left[{ }^{\circ} \mathrm{C}\right]$ & $7 \mathrm{CB}$ & $f_{1}$ & $f_{2}$ \\
\hline 30 & 0.116 & 0.135 & 0.155 \\
35 & 0.105 & 0.124 & 0.146 \\
40 & 0.094 & 0.114 & 0.135 \\
\hline
\end{tabular}

Table 1: The critical field of magnetic Fredericksz transition for $7 \mathrm{CB}$ based ferronematics

The influence of the concentration of 7CB based liquid crystals doped magnetic particles on the threshold field is summarized in Table 1. The influence of the concentration of doped magnetic particles on the threshold field for MBBA based ferronematics is given in Fig. 2. From the experimental resuts the increase of critical magnetic field in $7 \mathrm{CB}$ liquid crystal case is observed whereas decrease of critical magnetic field vs. volume concentration of magnetic particles in MBBA is found.

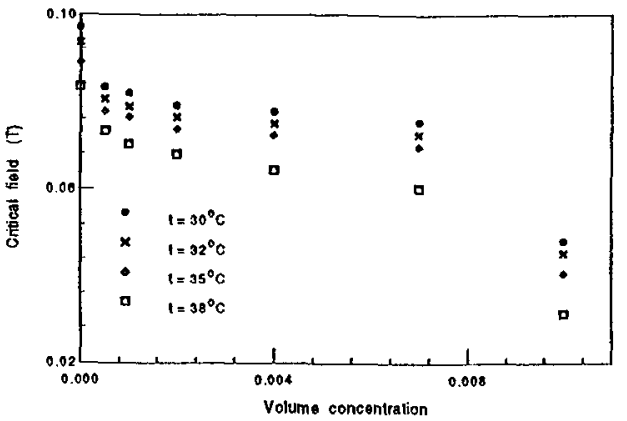

Figure 2: The critical magnetic field of Fredericksz transition vs volume concentration of magnetic particles for MBBA based ferronematics.

It is difficult to use the existing theories $[1,3,4]$ to our system because they are related to ferronematics with rod - like magnetite particles. In our system we have particles of nearly spherical shape. Qualitatively we can say that in $7 \mathrm{CB}$ system probably the initial condition $\vec{n}(\vec{r}) \perp \vec{m}(\vec{r})$ required from Burylov and Raikher condition $[3,4]$ is fulfiled whereas in MBBA is not. The co-aligment arrangement can take place in Burylov and Raikher theory provided W (surface density of anisotropic part of interfacial energy on the particle nematic boundary) is negative as discussed in paper [3]. After performing the analogous calculations scheme in co-alignment situation using the same approximations we obtain for critical magnetic field of Fredericksz transition the relation as

$H_{c}^{2}(f)-H_{c}^{2}(0)=2 W f / \chi_{a} d$

where $\chi_{a}$ is the anisotropy part of the nematic liquid carrier diamagnetic susceptibility, $d$ is size of magnetic particles and $H_{c}(0)=\left(\frac{\pi}{D}\right)\left(\frac{K}{\chi_{a}}\right)^{1 / 2}$, where $\mathrm{K}$ is elastic constant.

In conclusion we can say that MBBA - based ferronematic is probably a candidate with negative surface energy coupling between magnetic particles and the nematic liquid crystal molecules.

\section{Acknowledgment}

This work was supported by the Slovak Academy of Sciences within the framework of Project GAV No. 1361.

\section{References}

[1] Brochard F. and P.G. de Gennes, Le Journal de Physique 31 (1970) 691 - 708.

[2] Rault J, Cladis P.E. and Burger J.P., Phys. Letters 32A (1970) $199-200$.

[3] Burylov S.V. and Raikher Yu.L., J, Magn. Magn. Mater. 85 (1990) $74-76$.

[4] Burylov S.V. and Raikher Yu.L., J. Magn. Magn. Mater. 122 (1993) 62 - 65. 